\title{
Progress in Light Hadron Spectroscopy
}

\author{
Giulio Mezzadri ${ }^{* \dagger}$ \\ INFN Ferrara - University of Ferrara \\ E-mail: gmezzadrefe.infn.it
}

The BESIII experiment at the electron positron collider BEPCII in Beijing is successfully operating since 2008 and has collected large data samples in the tau-mass region, including the world largest data samples at the $J / \psi$ and $\psi$ resonances. In particular decays of these two resonances provide a rich and clean environment to study hadrons consisting out of light quarks and search for exotics. The BESIII collaboration has recently started a campaign to understand the nature of the $\mathrm{X}(1835)$ and $\mathrm{Y}(2175)$ resonances, which are debated to be exotic matter. Important observations have also been achieved in baryon spectroscopy, where the analyses benefit from the well defined initial state in $e^{+} e^{-}$collisions. Further, decays of $\eta$ mesons are studied to deepen our knowledge of their structure and possible symmetry breaking effects in their decays. In this presentation recent results of the light hadron physics program will be highlighted.

54th International Winter Meeting on Nuclear Physics

25-29 January 2016

Bormio, Italy

* Speaker.

${ }^{\dagger}$ on behalf of BESIII Collaboration 


\section{Introduction}

One of the crucial task at the present status of understanding of the nature of the Quantum Chromodynamics (QCD) is to understand the rich spectrum of particles at low energies. In that energy regime dwells the most interesting part of the QCD, where the perturbative approach does not held anymore. Moreover, confinement and hadronization are at full strength. Only with a deep understanding of this energy regime, will be possible to enlighten all the features of this theory.

The Beijing Electron Spectrometer III (BESIII) experiment at the electron positron collider Beijing Electron Positron Collider II (BEPCII) hosted at the Institute of High Energy Physics (IHEP) of Beijing has collected the world's largest sample of $J / \psi$ and $\psi^{\prime}$ since the start of its operation in 2008. The collider has recently reached the design luminosity of $10^{33} \mathrm{~cm}^{-2} \mathrm{~s}^{-1}$ : this will provide more data for the next future. With such a large sample, combined with the other data collected in DD thresholds and on other resonances (i.e. the Y(4260)), it is possible to complete the rich physics program [1], that covers not only light hadron physics, but also charmonium spectrum, open charm decays and exotic states.

The BESIII experiment [2] is composed by a series of concentric detectors, that allow the detection and the reconstruction of energy, charge and momentum of particles, and cover 93\% of the full solid angle. Just outside the beam pipe of beryllium, the He-based Multilayer Drift Chamber (MDC) is used to reconstruct charged particles with a momentum resolution at $1 \mathrm{GeV}$ of $\sigma_{p} / p=0.5 \%$ and energy resolution around 6\%. In the external part of the MDC, a series of Time-of-Flight detectors consisting of plastic scintillator counters provide the time information for particle identification (PID). Another crucial information for the PID is the energy of both charged and neutral tracks. A CsI $(\mathrm{Tl})$ electromagnetic calorimeter (EMC) is installed with a energy resolution for track at $1 \mathrm{GeV}$ of $\sigma_{E} / E=2.5 \% .1 \mathrm{~T}$ axial magnetic field is produced by a superconducting solenoid (SSM). In the return yoke of the magnet, Resistive Plate Chambers (RPCs) layers work as muon identifiers. A solid scheme of the detector it is shown in Fig. 1.

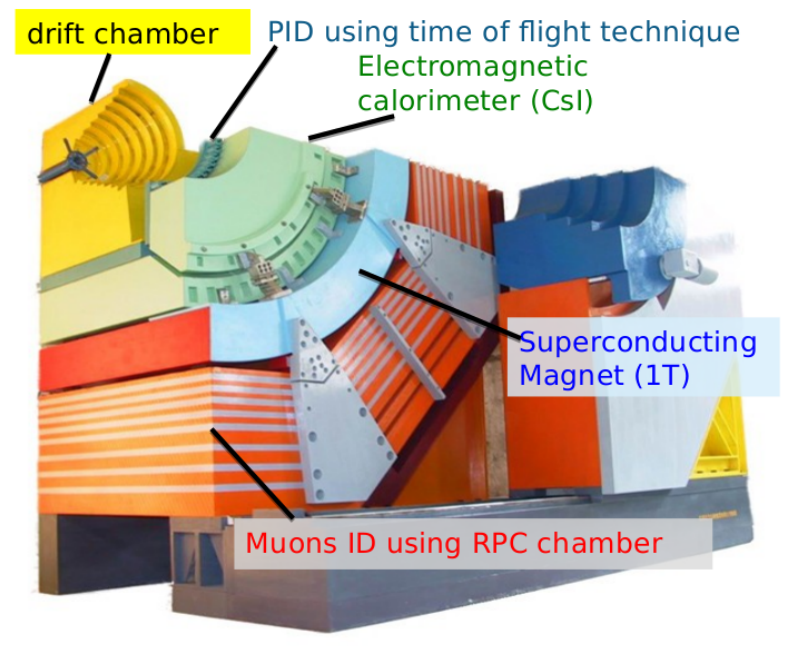

Figure 1: (color online) BESIII detector schematic drawings 
In this contribution, the attention will be focused on the progress in light hadron spectroscopy. The chosen topics are a part of a larger program in BESIII: a partial wave analysis (PWA) of the system $\pi^{0} \pi^{0}$ will be shown with a focus of the parametrization of the $\pi \pi$ scattering amplitude [3]; the latest result about the X(1835) state in BESIII will be shown [4], with particular attention on the determination of its spin-parity; the most recent BESIII discovery in baryon spectroscopy on $\Xi^{*}$ system will be briefly discussed [5].

\section{Spin-parity determination of $\mathrm{X}(\mathbf{1 8 3 5})$}

First discovered by BESII in the $J / \psi \rightarrow \gamma \eta^{\prime} \pi^{+} \pi^{-}$radiative decay [6] and later confirmed by BESIII [7], the X(1835) state nature has not be fully understood. Several hypothesis are discussed among theoretician: two models, the $p \bar{p}$ bound state [8] and the glueball candidate [9] are two possible explanations. BESIII has the opportunity to distinguish between the aforementioned hypotheses, using the world largest statistic of $J / \psi$ in the world. One way to disentangle is to perform a line shape study of the decay $J / \psi \rightarrow \gamma \eta^{\prime} \pi^{+} \pi^{-}$. BESIII has recently performed such study. [10] The results points towards two hypothesis: the first one describes the opening of a threshold, while the second one describes the line shape by means of a coherent sum of two BW amplitudes. Both model have comparable significance and supports the existence of two resonance. One other way possible way is to compare the branching ratio of the $\mathrm{X}(1835)$ in different final states. In this part of the contribution, the measurement of the branching ratio of the $\mathrm{X}(1835)$ in the decay mode $J / \psi \rightarrow \gamma K_{S} K_{S} \eta$ will be discussed. Given the large statistics and by means of a partial wave analysis, it is possible also to determine the spin-parity of the $\mathrm{X}(1835)$ candidate in this final state.

The decay mode analysed $J / \psi \rightarrow \gamma K_{S} K_{s} \eta$ shows several advantages to determine the $J^{P C}$ of the $\mathrm{X}(1835)$ : it is similar to the process in which this state was discovered; due to exchange symmetry and CP conservation, the main two sources of background, $J / \psi \rightarrow \pi^{0} K_{S}^{0} K_{S}^{0} \eta$ and $J / \psi \rightarrow$ $K_{S}^{0} K_{S}^{0} \eta$ are forbidden; the background is well known and the uncertainties due to its modeling are minimal.

The results of the selection is shown in Fig. 2(a). A clear $\eta_{c}$ signal is observed, and it is also possible to see a clear signal around $1.85 \mathrm{GeV} / \mathrm{c}^{2}$. From Fig. 2(b), it is possible to identify a $f_{0}(980)$ in $K_{S}^{0} K_{S}^{0}$ invariant mass; the scatter plot in Fig. 2(c) shows a strong correlation between the $1.85 \mathrm{GeV} / \mathrm{c}^{2}$ signal and the $f_{0}(980)$, as shown in Fig. $2(\mathrm{~d})$, where the selection on the $K_{S}^{0} K_{S}^{0}$ mass, i.e. $M_{K_{S}^{0} K_{S}^{0} \eta}<1.1 \mathrm{GeV} / \mathrm{c}^{2}$, is applied.

After all the aforementioned cut, since the sample is quite large, a PWA analysis was performed to enlighten the properties of the $1.85 \mathrm{GeV} / \mathrm{c}^{2}$ state. The restrictions on $M_{K_{S}^{0} K_{S}^{0} \eta}<2.8 \mathrm{GeV} / \mathrm{c}^{2}$ and $M_{K_{S}^{0} K_{S}^{0}}<1.1 \mathrm{GeV} / \mathrm{c}^{2}$ reduce complexities due to additional intermediate processes. Parity conservation restricts the possible $J^{P C}$ of the $K_{S}^{0} K_{S}^{0} \eta(\mathrm{X})$ system to be $0^{-+}, 1^{++}, 2^{++}, 2^{+-}, 3^{++}$. Only $J<3$ and possible $\mathrm{S}$-wave and P-wave decays are considered. PWA includes a $\mathrm{X}(1835)$ resonance in the $f_{0}(980) \eta$ and non-resonant component in one of the possible decay channels $f_{0}(980) \eta, f_{0}(1500) \eta$ or $f_{2}(1525) \eta$. The results are shown in Fig. 3. The contribution from $X(1835) \rightarrow K_{S}^{0} K_{S}^{0} \eta$ is required with a statistical significance greater than $12.9 \sigma$. The spin-parity is determined to be $0^{+-}$. The mass and the width are measured to be $1844 \pm 9(\text { stat })_{-25}^{+16}$ (syst $) \mathrm{MeV} / \mathrm{c}^{2}$ and $192_{-17}^{+20}(\text { stat })_{-43}^{+62}($ syst $) \mathrm{MeV}$ respectively. The determined branching fraction is measured to be $\left(3.31_{-0.3}^{+0.33}(\text { stat })_{-1.29}^{+1.96}(\right.$ syst $\left.)\right) \times 10^{-5}$. The observed values are consistent with the values of 

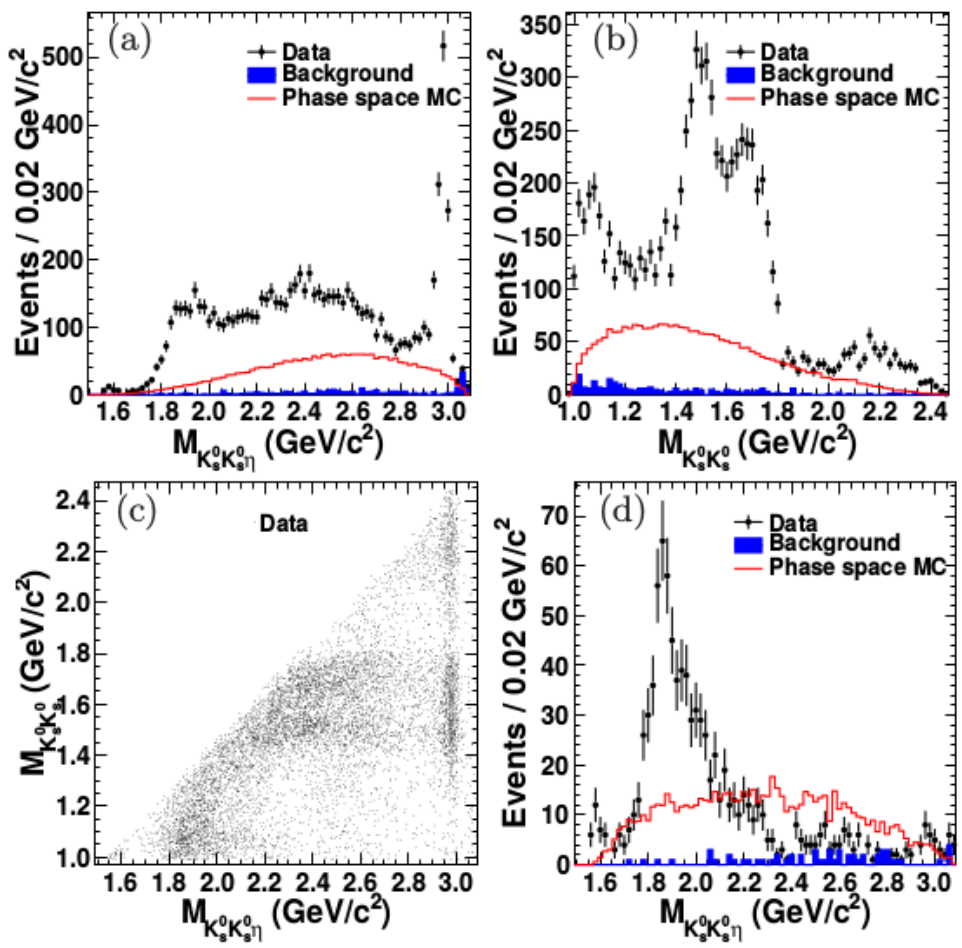

Figure 2: (color online) Fig. (a) shows the invariant mass distribution of $K_{S}^{0} K_{S}^{0} \eta$. The comparison between the pure phase space shows a clear $\eta_{c}$ signal and an enhancement around $1.85 \mathrm{GeV} / \mathrm{c}^{2}$. Fig. (b) shows the invariant mass of $K_{S}^{0} K_{S}^{0}$. The enhancement close to threshold can be identified with $f_{0}(980)$. Fig. (c) shows the Dalitz plot. A correlation between $f_{0}(980)$ and the enhancement at $1.85 \mathrm{GeV} / \mathrm{c}^{2}$ is seen. Fig. (d) shows the invariant mass of $K_{S}^{0} K_{S}^{0} \eta$, with $K_{S}^{0} K_{S}^{0}<1.1 \mathrm{GeV} / \mathrm{c}^{2}$. The enhancement grow larger as expected from the Dalitz plot shown in (c)

$J / \psi \rightarrow \gamma \eta^{\prime} \pi^{+} \pi^{-}$by BESIII. Another pseudo scalar states, the X(1560), is also observed in data with a statistical significance larger than $8.9 \sigma$ and is seen to interfere with the $\mathrm{X}(1835)$. The mass and the width are $1565 \pm 8(\text { stat })_{-63}^{+0}($ syst $) \mathrm{MeV} / \mathrm{c}^{2}$ and $45_{-13}^{+14}(\text { stat })_{-28}^{+21}($ syst $) \mathrm{MeV}$, respectively.

\section{Amplitude analysis of the $\pi^{0} \pi^{0}$ system in radiative $J / \psi$ decays}

The light pseudo scalar sector of QCD holds as one of the most complicated to be understood. The presence of large overlapping resonances and open threshold leads to a lack of understanding. The PDG [11] reports eight $I^{G} J^{P C}=0^{+} 0^{++}$mesons, which have widths between 100 and 450 $\mathrm{MeV}$. While the analysis is complicated, knowledge of this energy regime is crucial: in fact it is the most direct way to test the prediction of QCD models.

The scalar meson spectrum has been gone under test several times ([12], [13], [14]): an attractive technique using the K-matrix formalism has been performed using data from different production sources [15]. The study of radiative $J / \psi$ decay will give a benefit to similar analyses. Parity conservation in strong and electromagnetic decays restricts the quantum number accessible 


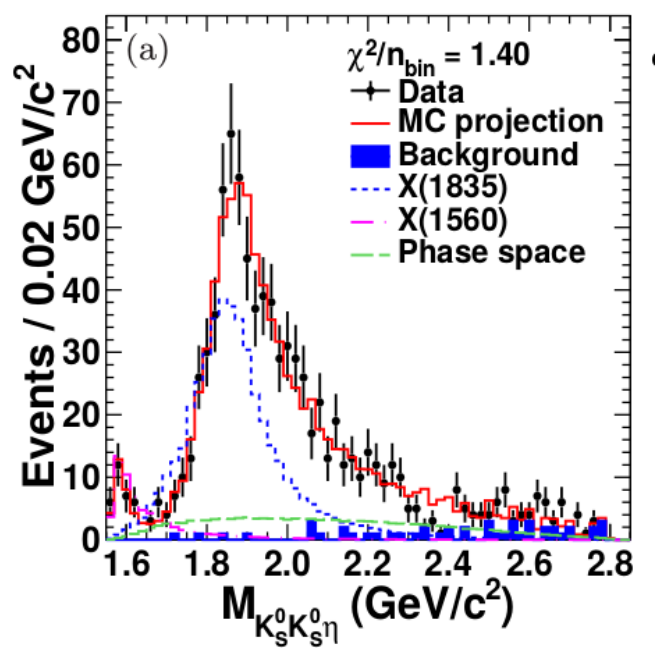

Figure 3: (color online) Invariant mass of $K_{S}^{0} K_{S}^{0} \eta$ is presented. The PWA requires both $\mathrm{X}(1835)$ and $\mathrm{X}(1560)$ contribution to fit the experimental data

and makes the PWA simpler than in other cases. The neutral channel $\left(\pi^{0} \pi^{0}\right)$ is of particular interest also due to the lack of sizable background like $\rho \pi$.

Taking profit of the world largest sample of $J / \psi$, this analysis want to test a different approach to the problem of PWA. Usually, a coherent sum of Breit-Wigner functions is build to model the $s$-dependence of $\pi \pi$ interaction [16]. This hypothesis would be proven true only in the limit of narrow resonances far from open thresholds. The idea instead is to measure the components of $\pi \pi$ amplitude independently for many small mass region. This allows to make minimal assumptions about the $s$-dependence of the $\pi \pi$ interaction.

In Fig 4 it is possible to see the $\pi^{0} \pi^{0}$ spectrum. The main background sources are extracted for inclusive montecarlo samples and are mainly composed by $\omega \pi^{0} \pi^{0}, b_{1} \pi^{0}, \gamma \eta\left(^{\prime}\right), \omega \pi^{0}$.

While the description of the model independent approach is beyond of the aim of this contribution, is it possible to discuss the most interesting results from Fig. 5. Significant structures in the $0^{++}$amplitude just below $1.5 \mathrm{GeV} / \mathrm{c}^{2}$ and near $1.7 \mathrm{GeV} / \mathrm{c}^{2}$ are clearly seen in the data. These states are believed to be collection of two scalar light quarks and a scalar glueball. Additional structures are present below $0.6 \mathrm{GeV} / \mathrm{c}^{2}$ and near $2.0 \mathrm{GeV} / \mathrm{c}^{2}$. The presence of four states below $2.1 \mathrm{GeV} / \mathrm{c}^{2}$ is consistent with previous BESII measurements [16]. Other two structure can be identified but with smaller enhancements. The $2^{++}$amplitude results are dominated by what appears to be the $f_{2}(1270)$. However, differences with a previous analysis [16] are present.

For the first time, it is also possible to extract the branching fraction of radiative $J / \psi$ decays into $\pi^{0}$ pairs, and it is measured to be $(1.15 \pm 0.05) \times 10^{-3}$.

\section{Measurements of $\psi(3686) \rightarrow K^{-} \Lambda \bar{\Xi}^{+}+$c.c. and $\psi(3686) \rightarrow \gamma K^{-} \Lambda \bar{\Xi}^{+}+$c.c.}

Being one of the best achievement of the last century, the quark model can provide a rather good description of the hadron spectrum. However, since many of the state predicted are undiscov- 


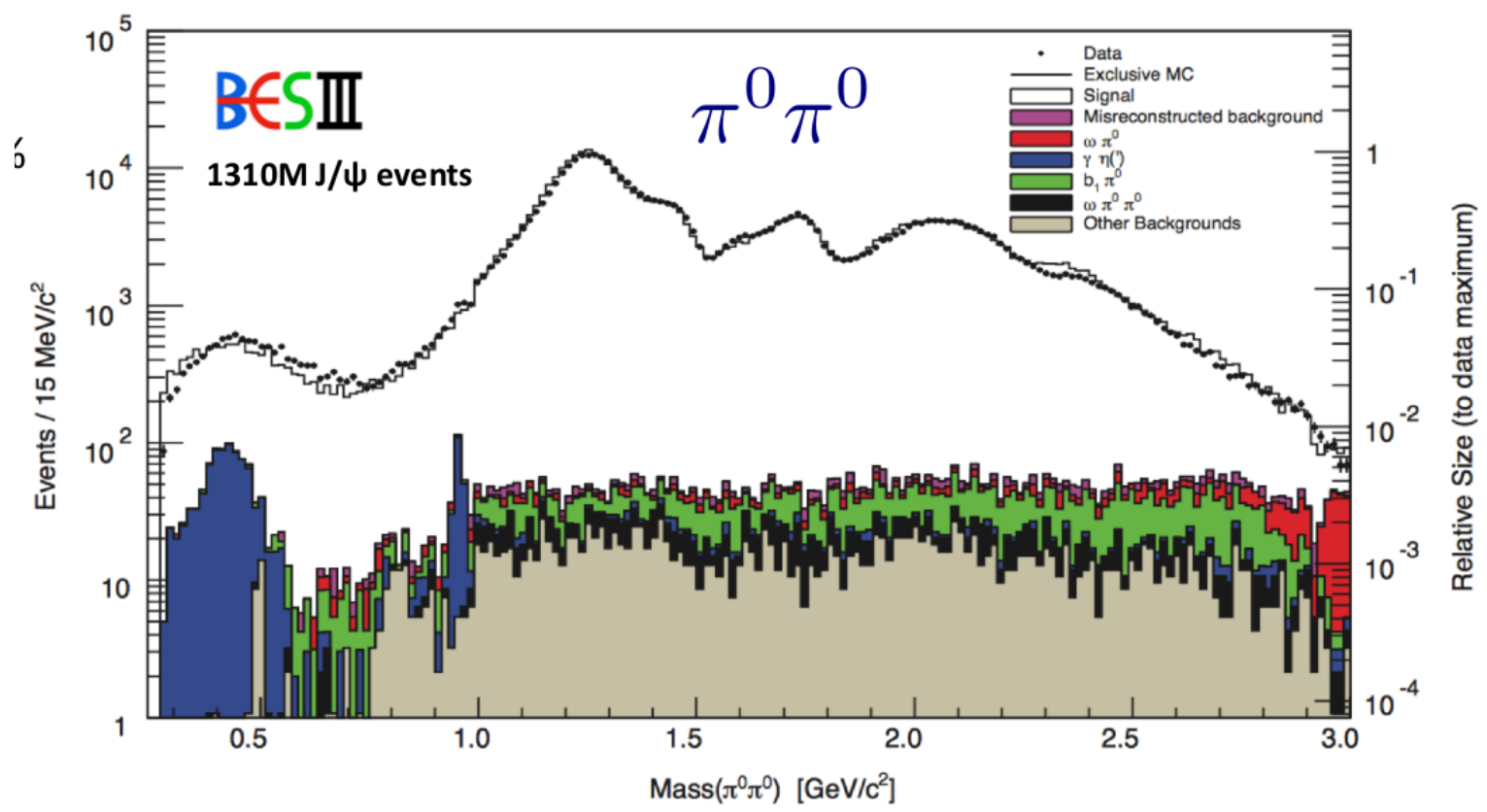

Figure 4: (color online) Invariant mass of $\pi 0 \pi 0$ with stacked histogram of the background contribution. The main contribution are given by $\left.\omega \pi^{0} \pi^{0}, b_{1} \pi^{0}, \gamma \eta{ }^{\prime}\right), \omega \pi^{0}$.

ered or not well established[27], it is far from being complete, especially in the case of states with $S=-2$.

In the following lines, the attention will be focused on the first measurements of the properties of two established hyperons, i.e. $\Xi(1690)$ and $\Xi(1820)$, in charmonium decays. These decays provide an ideal laboratory to study the hyperons. Despite the small phase space, the narrowness of the charmonia width reduces the overlap with neighboring states and the environment is clear from background.

As shown in Fig 6. a clear signal of both states is achievable in the $K^{-} \Lambda$ invariant mass in $\psi(3686) \rightarrow K^{-} \Lambda \bar{\Xi}^{+}$. Due to limited statistic is not possible to determine the spin-parity of the hyperons, so in the fit, these values are fixed to the previous experimental results([28], [29]). The fit results are shown in Tab. 1.

In the study of $\psi(3686) \rightarrow \gamma K^{-} \Lambda \bar{\Xi}^{+}$, several important results are achieved. As shown in Fig 7, a clear signal of $\psi(3686) \rightarrow K^{-} \Sigma^{0} \bar{\Xi}^{+}$is present in data: the determined branching fraction is measured to be $\mathscr{B}\left(\psi(3686) \rightarrow K^{-} \Sigma^{0} \bar{\Xi}^{+}\right)=(3.67 \pm 0.33) \times 10^{-5}$, where the errors are statistical only. It is possible to determine the yields of $\chi_{c J} \rightarrow K^{-} \Lambda \bar{\Xi}^{+}(J=0,1,2)$ by fitting the invariant mass distribution of $K^{-} \Lambda \bar{\Xi}^{+}$. The results are shown in Fig 8 and the branching fractions $\mathscr{B}\left(\psi(3686) \rightarrow \gamma \chi_{c J}\right) \times \mathscr{B}\left(\chi_{c J} \rightarrow K^{-} \Lambda \bar{\Xi}^{+}\right)$are determined to be $(1.90 \pm 0.30) \times 10^{-5}$, $(1.32 \pm 0.20) \times 10^{-5}$ and $(1.68 \pm 0.26) \times 10^{-5}$ for $\chi_{c J}(J=0,1,2)$ respectively.

\section{References}

[1] D. M. Asner et al., Physics at BES-III, arXiv:0809.1869v1 [hep-ex] (2008) 

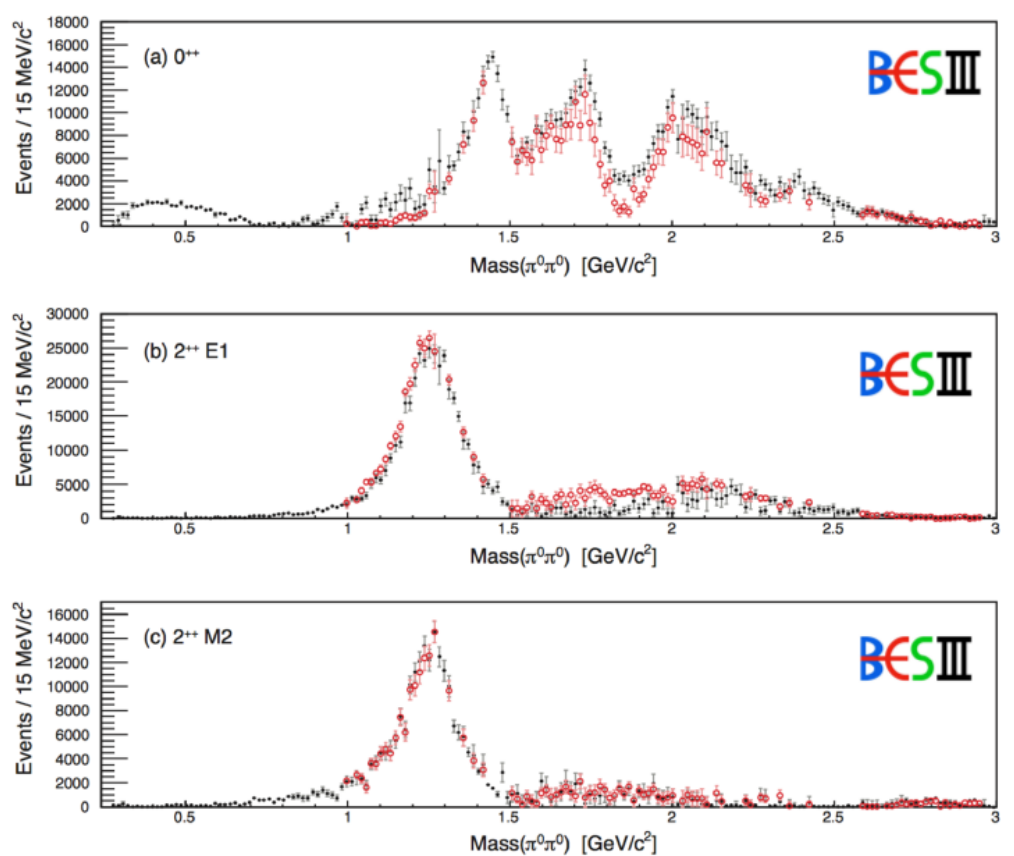

Figure 5: (color online) Results for the model independent partial wave analysis. The black dots represents th intensity calculated from one set of solution, while the red ones represents the ambigous partner. Only statistical errors are presented.

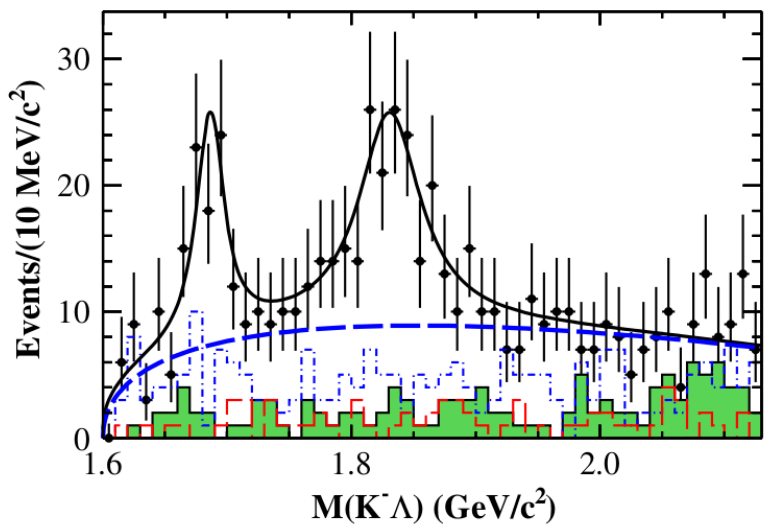

Figure 6: (color online) Invariant mass of $K \Lambda$. Clear signal of $X i(1690)$ and $\Xi(1820)$ are identificable.

[2] M. Ablikim et al. (BESIII Collaboration), Nucl. Instrum. Meth. A 614, 345 (2010)

[3] M. Ablikim et al. (BESIII Collaboration), Phys. Rev. D 92, 052003 (2015)

[4] M. Ablikim et al. (BESIII Collaboration), Phys. Rev. Lett. 115, 091803 (2015)

[5] M. Ablikim et al. (BESIII Collaboration), Phys. Rev. D 91092006 (2015)

[6] M. Ablikim et al. (BES Collaboration), Phys. Rev. Lett. 95, 262001, (2005). 


\begin{tabular}{|ccc|}
\hline \hline & $\Xi(1690)^{-}$ & $\Xi(1820)^{-}$ \\
\hline $\mathrm{M}\left(\mathrm{MeV} / \mathrm{c}^{2}\right)$ & $1687.7 \pm 3.8 \pm 1.0$ & $1826.7 \pm 5.5 \pm 1.6$ \\
\hline$\Gamma(\mathrm{MeV})$ & $27.1 \pm 10.0 \pm 2.7$ & $54.4 \pm 15.7 \pm 4.2$ \\
\hline Significance $(\sigma)$ & 4.9 & 6.2 \\
\hline $\mathrm{B}\left(10^{-6}\right)$ & $5.21 \pm 1.48 \pm 0.57$ & $12.03 \pm 2.94 \pm 1.22$ \\
\hline$M_{P D G}\left(\mathrm{MeV} / \mathrm{c}^{2}\right)$ & $1690 \pm 10$ & $1823 \pm 5$ \\
\hline$\Gamma_{P D G}(\mathrm{MeV})$ & $<30$ & $24_{-10}^{+15}$ \\
\hline \hline
\end{tabular}

Table 1: Results from the fit at the signal shown in Fig 7. For more information about the fit please refer to the main article and reference therein
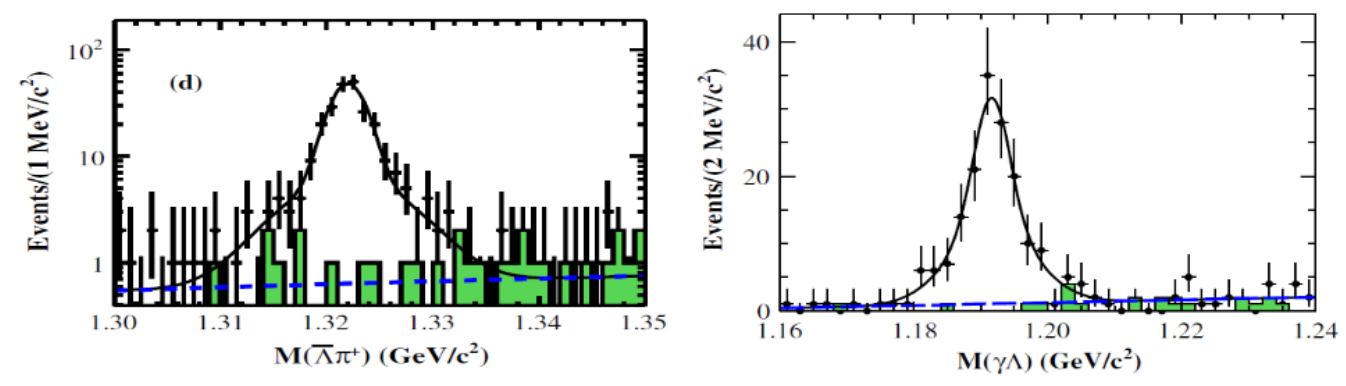

Figure 7: (color online) Invariant mass distribution of $\gamma \Lambda$ in $\psi(3686) \rightarrow \gamma K^{-} \Lambda \bar{\Xi}^{+}$. A clear signal of $\sigma 0$ is seen.

[7] M. Ablikim et al. (BESIII Collaboration), Phys. Rev. Lett. 106, 072002 (2011).

[8] Z.G. Wang, Chin. Phys. Lett. 27101201 (2010).

[9] B.A. Li, Phys. Rev. D 74034019 (2006).

[10] M. Ablikim et al. Phys. Rev. Lett. 117, 042002 (2016).

[11] K.A. Olive et al. (Particle Data Group), Chin. Phys. C, 38, 090001 (2014 and 2015 update).

[12] J. Gunter et al (E852 Collaboration), Phys. Rev D, 64, 072003 (2001)

[13] A. Abele et al. (Crystal Barrel Collaboration), Phys. Lett. B 380, 296 (1999)

[14] R. Bellazzini et al. (GAMS Collaboration), Phys. Lett. B, 645, 19 (2007)

[15] V. Anisovich and A. Sarantsev, Eur. Phys. J. A 16, 229 (2003)

[16] M. Ablikim et al. (BES Collaboration), Phys. Lett. B 642, 441 (2006)

[17] L.W. Bartel et al. (JADE Collaboration), Phys. Lett. B 113, 190 (1982)

[18] H. Behrends et al. (CELLO Collaboration), Phys. Lett. B 114, 78 (1982)

[19] C. Berger et al (PLUTO Collaboration), Phys. Lett. B 142, 125 (1984)

[20] M. Althoff et al. (TASSO Collaboration), Phys. Lett. B 147, 487 (1984)

[21] H. Aihara et al. (TPC/ $\gamma \gamma$ ), Phys. Rev D 35, 2650 (1987)

[22] H. Albrecht et al. (ARGUS Collaboration), Phys. Lett. B 199, 457 (1987) 


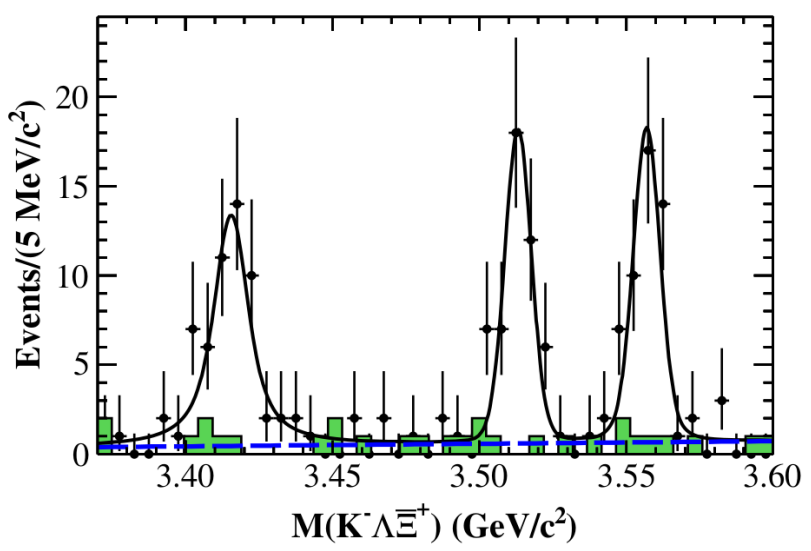

Figure 8: (color online) Invariant mass of $K \Lambda \Xi$ in $\psi(3686) \rightarrow \gamma K^{-} \Lambda \bar{\Xi}^{+}$. Signal of $\chi_{c J}(J=0,1,2)$ are clearly identificable.

[23] J. Wess and B. Zumino, Phys. Lett B 37, 95 (1971); E. Witten, Nucl Phys. B 223, 422 (1983).

[24] G.J. Gounaris, J.J. Sakurai, Phys. Rev. Lett 21, 244 (1968)

[25] J.P. Lees et al (BABAR Collaboration), Phys. Rev D 86, 032013 (2012)

[26] F. Stollenwerk, C. Hanhart, A. Kupsc, U.G. Meiner, A. Wirzba, Phys. Lett B707, 184 (2012)

[27] R. Horgan, Nucl. Phys. B71, 514, (1974); M. Jones, R.H. Dalitz and R. Horgan, Nucl. Phys. B129, 45, (1977)

[28] B. Aubert et al (BABAR Collaboration), Phys. Rev. D 78, 034080 (2008)

[29] J.B Gay et al, Phys. Lett. B 62, 477 (1976) 